\title{
Bringing the HEET: The Argument for High-Efficacy Early Treatment for Pediatric-Onset Multiple Sclerosis
}

\author{
Marisa McGinley $^{1} \cdot \operatorname{Ian}$ T. Rossman ${ }^{2}$
}

Published online: 11 September 2017

(C) The American Society for Experimental NeuroTherapeutics, Inc. 2017

\begin{abstract}
Pediatric-onset multiple sclerosis (POMS) is rarer than adult-onset disease, and represents a different diagnostic and treatment challenge to clinicians. We review POMS clinical and radiographic presentations, and explore important differences between POMS and adult-onset MS natural histories and long-term outcomes. Despite having more active disease, current treatment guidelines for patients with POMS endorse the off-label use of lower-efficacy disease-modifying therapies (DMTs) as first line. We review the available MS DMTs, their evidence for use in POMS, and the contrasting treatment strategies of high-efficacy early treatment and escalation therapy. We introduce a new treatment approach, the "high-efficacy early treatment", or HEET strategy, based on using directly observed, high-efficacy intravenously infused DMTs as first-line therapies. Like other proposed POMS treatment strategies, HEET will need to be prospectively studied, and all treatment decisions should be determined by an experienced neurologist, the patient, and his/her parents.
\end{abstract}

Keywords Pediatric-onset multiple sclerosis . disease-modifying therapy $\cdot$ neurodevelopment . natalizumab $\cdot$ rituximab $\cdot$ NEDA

Electronic supplementary material The online version of this article (https://doi.org/10.1007/s13311-017-0568-1) contains supplementary material, which is available to authorized users.

Ian T. Rossman

irossman@chmca.org

1 Mellen Center for Multiple Sclerosis Treatment and Research, Cleveland Clinic, 9500 Euclid Avenue U10, Cleveland, OH 44195, USA

2 NeuroDevelopmental Science Center, Akron Children's Hospital, One Perkins Square, Akron, OH 44308, USA

\section{Introduction}

While most commonly presenting in young adults, approximately $5 \%$ of patients with multiple sclerosis (MS) experience onset of symptoms before 18 years of age, defined as pediatric-onset MS (POMS). In the USA, studies estimate a yearly incidence of POMS between 0.18 and $0.51 / 100,000$, though the true worldwide incidence of POMS is not known and likely varies by ethnicity and geography $[1,2]$. POMS presents both a diagnostic and treatment challenge for several reasons. Recognition and diagnosis of POMS is often delayed as pediatricians and pediatric neurologists may not be familiar with pediatric demyelinating disease symptoms and signs. Further complicating matters are the differences in presentation and differential diagnoses between patients with pre- and postpubertal POMS. Even when diagnosed appropriately, there are no approved POMS treatments in the USA. Thus, parents and children with POMS are faced with a rare diagnosis, a paucity of information, a complete lack of approved treatment options, and a lot of uncertainty about their futures.

Consensus diagnostic criteria, treatment guidelines, and research studies have emerged to enhance our recognition and understanding of POMS, and to improve our treatment of this disease $[3,4]$. Through these efforts we are better able to inform parents, patients, and clinicians about the important differences and similarities between POMS and adult-onset MS. As we will review, compared with adult patients with MS, patients with POMS demonstrate more inflammatory demyelination early in their disease, resulting in increased clinical relapses, rapid MRI lesion accrual, early inflammatory tissue-level injury, and, ultimately, early disability progression. Despite these important differences, current POMS treatment strategies tend to favor lower-efficacy disease-modifying therapies (DMTs), whereas current adult regimens move toward higher-efficacy DMTs. 
While community awareness and research efforts have grown tremendously in the last decade, there remains a huge disparity between treatment options for POMS and adult MS. As reviewed in this issue of Neurotherapeutics, practitioners in the field of adult MS increasingly recognize the importance of early treatment with high-efficacy DMTs to halt MS disease activity and prevent permanent injury to the central nervous system (CNS). Concepts such as "no evidence of disease activity" (NEDA), defined as no clinical relapses, no new or enlarging T2 hyperintense lesions or gadolinium-enhancing T1 lesions on MRI, and no disability progression are no longer merely research concepts but rather guide clinical decisionmaking. However, there is a dearth of pediatric pharmacokinetic, pharmacodynamic, safety, tolerability, and efficacy trials in general, and of MS DMTs in particular [5]. The scarcity of patients with POMS, as well as the status of children as a vulnerable population makes large-scale trials of MS DMTs in pediatric populations difficult [6]. Recently pharmaceutical companies were incentivized and required by the US government to include pediatric clinical trials in their pipeline for existing drugs [5]. However, these studies have been slow to enroll and ethical issues remain regarding primary endpoints, study duration, and the use of placebo versus active comparator drugs.

Current treatment guidelines for POMS are comprised of expert opinion based on available retrospective observational, case series, and, in some cases, prospective safety data. The current POMS treatment guidelines focus on a stepwise therapy escalation, but it is the authors' opinion that higherefficacy therapies should be considered at the time of diagnosis. This paper will review some of the unique aspects of diagnosis, clinical presentation, imaging, and natural course of POMS that highlight the need to consider high-efficacy treatments early. Additionally, several important differences between pre- and postpubertal POMS will be discussed to justify why it might be important to modify treatment regimens based on pubertal status. Recognizing that POMS is simultaneously a neurodevelopmental, inflammatory demyelinating, and neurodegenerative disease, the treatment recommendations presented here aim to improve POMS outcomes by preventing irreversible neurologic impairment. It is our hope that our "high-efficacy early treatment" (HEET) strategy presented here will be considered for prospective study in patients with POMS and updated to reflect the safest, most efficacious treatment options available.

\section{Diagnosis}

POMS is one of a family of acquired inflammatory demyelinating conditions affecting the pediatric population, including acute disseminated encephalomyelitis (ADEM), MS, and neuromyelitis optica spectrum disorder. The International
Pediatric Multiple Sclerosis Study Group recently updated the diagnostic criteria for POMS in 2016 [3, 4]. As in adult patients, POMS diagnosis relies on clinically identifiable neurologic symptoms and signs, and radiographic evidence of demyelinating lesions. These clinical and radiographic findings must satisfy dissemination in time and dissemination in space, as defined by the 2010 Revised McDonald Criteria [7].

The 2010 Revised McDonald criteria have been studied in children older than 11 years and have a reported positive predictive value of $76 \%$ and negative predictive value of $100 \%$ [8]. However, for children $<12$ years old, the positive predictive value for the 2010 criteria was only $55 \%$, which illustrates one of the many quantitative differences between pre- and postpubertal patients with POMS [8]. One of the unique clinical aspects of POMS is the need to address if, and when, an episode of ADEM should be utilized for an MS diagnosis. The 2016 revision of the International Pediatric Multiple Sclerosis Study Group 2013 proposed criteria for POMS diagnosis again allow ADEM to be the first clinical presentation of POMS as long as it is followed by a nonencephalopathic clinical event 3 or more months after symptom onset, and is associated with new magnetic resonance imaging (MRI) lesions fulfilling 2010 Revised McDonald Criteria [4, 7].

\section{Clinical Presentation}

The clinical features of POMS largely overlap with adultonset MS, including optic neuritis, sensory deficits, motor deficits, brainstem syndromes, cerebellar dysfunction, and spinal cord syndromes. A clinical history is often difficult to obtain in young children, and may rely on parental interpretation or observation. Similarly, adolescents and young adults are often not forthcoming about clinical symptoms, particularly those involving bladder or bowel dysfunction. Thus, a paucity of clinical details may delay diagnosis, or make demyelinating disease less obvious. For all postpubertal patients, clinicians ought to interview patients with and without a caretaker present to allow a more thorough history to be obtained. Prepubertal-onset MS is more likely to be a polysymptomatic severe first attack but with milder residual neurological sequelae. This presentation is in contrast to a postpubertal cohort that is more likely to have a monosymptomatic presentation with optic neuritis or sensory deficits [9], similar to adult presentations. Other important clinical differences in patients with POMS include more rapid recovery from clinical relapses compared with adults, with or without acute treatment, but with a higher relapse rate than patients with adult-onset MS (see section on natural history below). Alternatively, prior clinically isolated syndromes may have been mild or brief, and not come to the attention of a medical professional. 


\section{Imaging Criteria}

MRI is invaluable in the diagnosis of POMS (reviewed by Banwell et al [10]), and in patients 12 years and older may confirm POMS with a single study. A unique finding is that posterior fossa lesions are more common in children, and are typically a higher proportion of the overall lesion burden in POMS versus adult MS. In keeping with their increased frequency of clinical relapses, children are also more likely to have new T2 hyperintense and gadolinium-enhancing T1 lesions on follow-up scans after a clinically isolated syndromes, demonstrating that POMS typically presents with a more active inflammatory early disease course than in adults [11].

MRI findings in prepubertal POMS can also be dissimilar to those of postpubertal and adult-onset MS. Early-onset MS (median age 8.9 years) had fewer well-defined ovoid T2 bright lesions and more often had confluent lesions on their first MRI than patients with later-onset pediatric MS (median age 14.47 years) [12].

\section{Ancillary Testing: Cerebrospinal Fluid Profile}

In addition to clinical and radiographic criteria, cerebrospinal fluid (CSF) examination may be necessary to help differentiate POMS from other diagnoses. A recent study indicated that the presence of oligoclonal bands in the CSF at the time of an initial clinical attack doubles the risk of having a second attack [13]. In children, these findings may also be supportive, but younger children (onset $<11$ years) have been shown to have a unique CSF profile. Pediatric patients with early-onset MS tend to have a higher white blood cell count with a higher proportion of neutrophils than patients with later-onset MS [14]. Additionally, fewer patients with early-onset MS had an elevated IgG index [14]. There is no consensus on the use of lumbar puncture for CSF analysis in the diagnosis of POMS, and clinical practice varies between practitioners. In the authors' practice, for postpubertal patients satisfying 2010 diagnostic criteria, CSF analysis is not needed. However, for any encephalopathic patient, febrile patient with focal neurologic deficits, developmentally delayed patient, or prepubertal patient in whom POMS is considered, CSF analysis is likely warranted.

\section{Natural History and Course}

The natural history and course of POMS is similar to adults, but there are some unique aspects that should be considered and may affect how we recommend approaching treatment. In a large, multicenter, observational study utilizing the European Database for Multiple Sclerosis, a cohort of 395 patients younger than 16 years was analyzed [15]. The mean age of onset in this cohort was 13.7 years and the initial course was relapsing remitting for $97.7 \%$. The median time between the first 2 neurological episodes was 2.0 years, whereas $28.6 \%$ converted to a secondary progressive course with mean time to conversion of 28.1 years (median age 41.4 years). On average, patients with POMS reached irreversible disability 10 years younger than patients with adult-onset MS [15]. Progression of cognitive problems in POMS is a significant concern because the inflammation, demyelination, and axonal damage is occurring in a still-developing CNS. In a 5-year study evaluating neuropsychological features in patients with POMS, $38 \%$ of subjects at year 5 fulfilled criteria for cognitive impairment, and deterioration was observed in $56 \%$ of patients, suggesting patients with POMS are particularly susceptible to cognitive problems [16]. Cognitive impairment is also common in adult MS. However, adults previously diagnosed with POMS demonstrate more severe impairment compared to age-matched adult-onset patients, even when adjusting for disease duration [17]. There have been several other observational studies that have focused on the relapse rate in POMS. A shorter interval duration between first and second relapses and a higher relapse rate were demonstrated in a younger cohort $(<15$ years) [18]. Two more recent observational studies, a 12-month and a longer 6-year study both found annualized relapse rates were significantly higher in patients with POMS, even when controlling for treatment with DMTs [19, 20]. Disability measured by the Expanded Disability Status Scale (EDSS) was similar across these studies. The observation that POMS has an increased relapse rate suggests it may have more active inflammation early in the disease course than in adult-onset disease. This clinical and radiological observation was supported by histological analysis of biopsied and autopsied pediatric and adult demyelinating lesions. Indeed, pediatric MS lesions were more likely to be early active demyelinating lesions with extensive axon loss compared, particularly in prepubertal patients, with postpubertal and adult patient lesions [21]. Further, the degree of axon loss was positively correlated with macrophage infiltration but not other autoreactive lymphocyte types. This tissue-level analysis demonstrates the early inflammatory and destructive nature of POMS-related inflammatory demyelinating disease [21]. Thus, the disconnect between highly destructive early inflammatory disease in POMS but delayed disability accrual likely reflects the "neurologic reserve" or plasticity of younger, developing brains. This may also shed light on the dual nature of MS as both an early inflammatory disease that transitions to a neurodegenerative disease in the later stages.

Similar to other chronic pediatric neurologic diseases, POMS represents a neurodevelopmental disorder with lifelong consequences. Longitudinal MRI studies demonstrate reduced age-expected whole brain, thalamic, and cerebellar growth in patients with POMS compared with age-matched controls [22-24]. Whole-brain and thalamic volume reduction 
is correlated with cognitive impairment, slower processing speed and inattention in a small cohort of patients with POMS compared with age-matched controls [25]. As stated above, patients with POMS have more posterior fossa lesion burden than adults, and posterior cerebellar volumes and posterior fossa lesion burden have been correlated with decreased cognitive function in patients with POMS [26]. Mathematical performance is also found to be impaired in patients with POMS versus age-matched controls, which correlates to white matter microstructural deficits identified by diffusion tensor imaging [27]. Functional MRI has also demonstrated decreased resting state functional connectivity between multiple brain regions in patients with POMS [28]. Patients with POMS also demonstrate social cognitive impairment not explained by deficits in processing speed [29]. Similar to adults, the psychosocial impact of POMS is multifactorial, with fatigue, depression, and physical disability all contributing [30]. However, psychosocial factors unique to children include negative impact of MS on their school performance and parental relationships [31, 32]. As adults, patients with POMS show more cognitive impairment, and achieve physical disability at a younger age than age-matched adult-onset patients with MS $[17,33]$. Thus, at a time when brain maturation and growth should be occurring, patients with POMS demonstrate neurodevelopmental and neurodegenerative changes, resulting in life-long functional consequences. Potentially, early treatment may prevent or slow some of these changes, leading to improved cognitive and psychosocial outcomes for patients with POMS.

\section{Treatment}

There are no Food and Drug Administration (FDA) approved DMTs to treat MS in patients under 18 years of age. Thus, pediatric neurologists have used DMTs off label to treat patients with POMS since their introduction in the early 1990s. As detailed below, current treatment recommendations for POMS are based on case series and reports and retrospective studies demonstrating tolerability and apparent efficacy of a DMT in children under 18 years old (see Table 1). There are several clinical trials recently completed or actively enrolling to demonstrate the safety, tolerability, and efficacy of existing DMTs in pediatric patients.

There are treatment challenges inherent to pediatric medicine not encountered when treating adult patients with MS. Compared with adult patients, patients with POMS have developing immune systems with more intact thymic tissue, and the impact of DMTs on immunodevelopment is largely unknown. Studies of POMS T-cell ratios demonstrate T-cell profiles more similar to adult control patients, suggesting that early thymic involution, reduced naïve $\mathrm{T}$ cells, and reduced regulatory T-cell receptor repertoire may be part of the pathogenesis of POMS [34]. Children's body weight and surface area can potentially drastically change during treatment, altering the pharmacokinetics of a DMT; prepubertal to postpubertal changes could also alter MS disease activity and response to DMT. There are complex social and emotional relationships during childhood and adolescence that complicate chronic disease management, and depending on the age at diagnosis, patients with POMS have varying amounts of autonomy over treatment choices. There are also long-term cost and side effect potentials for patients with POMS who will likely be on DMTs for longer than most patients with adult-onset MS. One of the most important differences from adult-onset MS is POMS represents a neurodevelopmental disease in addition to a neuroinflammatory and neurodegenerative disease. Thus, treatments that are well tolerated but inadequately protect the developing CNS from MS disease activity may be insidiously more harmful than those perceived to be "high risk". Thus, risk versus benefit strategies that may be straightforward in adult populations are more complex when considering treatment options for patients with POMS.

\section{Injectable DMTs}

The first DMTs to obtain FDA approval for the treatment of relapsing remitting MS (RRMS) in adults were all injectable medications. While injectable medication is generally avoided where possible in pediatric medicine, injectable DMTs were the first treatments available, thus they have the longest track record of use and are the most studied in POMS. These DMTs differ in their mechanisms of action, routes of administration (subcutaneous $v s$ intramuscular), and their dosing frequency from daily to once monthly.

Interferon (IFN)- $\beta 1 \mathrm{a}$ and IFN- $\beta 1 \mathrm{~b}$ (together will be referred to as "IFNs") likely have multiple effects on the immune system, ultimately promoting anti-inflammatory actions on $\mathrm{T}$ and $\mathrm{B}$ lymphocytes, antigen presenting cells and downregulating proinflammatory cytokines [35]. IFNs were the first to be investigated in a pediatric population through several retrospective and open-label, uncontrolled prospective studies [36-42]. All of these studies have provided evidence of IFN safety and tolerability, though pharmacokinetic and pharmacodynamics studies were not undertaken, and pediatric dosing was not established. Side effect profiles are similar to those seen with adults, including injection site reactions, flulike symptoms, liver function elevations, headache, and depression. Although most of these studies were not designed to evaluate efficacy, several demonstrated a reduction in annualized relapse rate [36-38, 41]. In one of these studies, the effect on annualized relapse rate was most significant during the first 2 years but less significant at 4 years [38]. The effect on disability progression in POMS has not been well established.

Glatiramer acetate likely works by inducing the T helper 2 suppressor phenotype and anti-inflammatory cytokine 


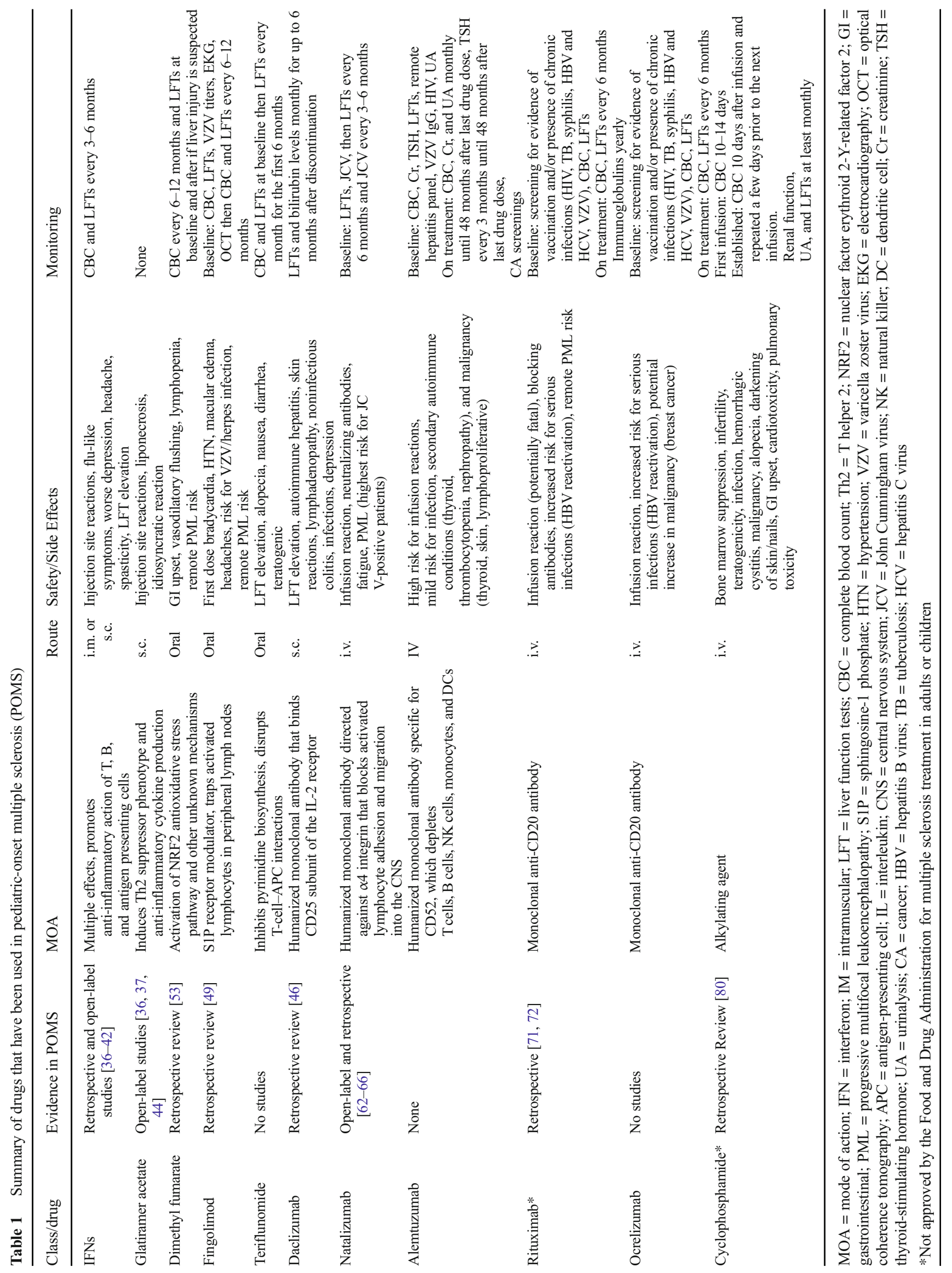


production [43]. Similar to the IFNs, several small open-label studies have demonstrated safety, tolerability, and clinical efficacy of glatiramer acetate in patients with POMS [36, 37, 44]. In contrast to other DMTs, glatiramer does not require interval blood draws for safety monitoring laboratories, making it easier to use in young children in whom venipuncture may be anxiety provoking.

Daclizumab is a humanized monoclonal antibody previously used as an antirejection medication following transplant that was recently approved for use in RRMS. This antibody against the CD25 (alpha) subunit prevents interleukin-2 binding high affinity interleukin-2 receptors, thereby reducing activation of autoreactive $\mathrm{T}$ lymphocytes and upregulating selftolerant CD56 $6^{\text {bright }}$ natural killer cells [45]. There is 1 openlabel trial of 7 pediatric patients utilizing intravenous daclizumab mostly in combination with either an IFN or glatiramer acetate [46]. This study did not use the currently approved daclizumab dose or route of administration, but overall did demonstrate modest clinical and radiographic benefit, as well as safety and tolerability. However, daclizumab requires close monitoring for adverse events, including severe transaminitis, severe skin reactions, infections, oral ulcers, and psoriasis. Daclizumab's subcutaneous route of administration, potential for severe side effects, required risk evaluation and mitigation strategy monthly laboratory testing, and modest clinical benefits compared with IFNs make this DMT less desirable for use in a pediatric population, which is also reiterated by the FDA label [47].

\section{Oral DMTs}

Since 2010 three oral medications have been approved for treatment of RRMS in adults, but to date there are limited data to support their use in POMS. Fingolimod was the first oral medication approved to treat RRMS. It is a sphingosine 1phosphate receptor modulator that sequesters lymphocytes in the peripheral lymphoid tissue [48]. To date there is only 1 published retrospective review of fingolimod in a pediatric population [49]. In this study 17 patients were identified in a Brazilian database. The average age was 16.1 years and 4 patients were treatment naïve, whereas the other 13 had a suboptimal response to at least 1 previous injectable therapy. Only 1 patient had a relapse, although the average follow-up in the study was short (8.6 months). The drug was well tolerated with no serious adverse events reported. The minimum heart rate during first dose was 52 beats and all patients returned to baseline after $6 \mathrm{~h}$ of monitoring. The lowest lymphocyte count observed was $456 / \mathrm{mm}^{3}$ and all other patients maintained lymphocyte counts $>500 / \mathrm{mm}^{3}$. There were no ophthalmological complications observed. Several infections were seen, including 1 case of genital herpes, 1 episode of upper airway viral infection, and 2 episodes of urinary tract infection. This study provides class IV evidence that fingolimod is potentially safe and tolerable in POMS. There is an ongoing Novartis-sponsored clinical trial (NCT01892722) to formally evaluate safety and efficacy of fingolimod in children [50]. Two considerations regarding fingolimod use in POMS include the risk of rebound MS disease activity following cessation of treatment or switching to a lower-efficacy DMT [51], and the risk for progressive multifocal leukoencephalopathy (PML), which will be discussed more fully in the section on natalizumab. As of 28 March 2017 there have been 10 confirmed PML cases in adult patients with MS receiving fingolimod (MM, personal communication with Novartis).

The second oral medication approved in adults is dimethyl fumarate, which activates nuclear factor erythroid 2-Y-related factor 2 in the antioxidative stress pathway, but the direct mechanism of dimethyl fumarate in MS treatment is unknown [52]. There is 1 retrospective review of 13 children (median age 13.08 years) who were treated with dimethyl fumarate either as a first- or second-line agent [53]. The side effects were consistent with the adult literature and included facial flushing, gastrointestinal discomfort, rash, and malaise. This study reported stabilization or improvement in both relapse rate and EDSS in all children, except 1 . Of the 13 children in the study, 10 tolerated the adult dose of $240 \mathrm{mg}$ twice daily and 3 only tolerated the $120 \mathrm{mg}$ twice-daily dose. The children who took the lower dose still had a beneficial effect in both EDSS and relapse rate, suggesting a lower dose might be efficacious in children. There was a higher rate of treatment discontinuation (23\%) than in the adult trials (12-16\%) [53]. There is currently a phase III clinical trial (CONNECT; NCT02283853) sponsored by Biogen to formally evaluate safety and efficacy of dimethyl fumarate in pediatric patients [54]. As with fingolimod, there have been cases of PML reported for patients taking dimethyl fumarate. As of 28 March 2017 there have been 6 confirmed PML cases in adult patients with MS receiving dimethyl fumarate (MM, personal communication with Biogen).

Teriflunomide is the third oral medication approved for RRMS in adults. It inhibits the mitochondrial enzyme dihydroorotate dehydrogenase, which is important for de novo pyrimidine synthesis; disrupting this pathway is thought to disrupt T-cell-antigen presenting cell interactions and T-cell division [55]. Currently, there are no published studies evaluating the safety or efficacy of teriflunomide in POMS, though an ongoing, randomized, phase III, placebo-controlled trial (TERIKIDS; NCT02201108) is currently enrolling, which aims to assess efficacy, safety, and pharmacokinetics of teriflunomide in POMS [56].

\section{Intravenous Infusions}

Natalizumab is a humanized monoclonal antibody directed against $\alpha 4$ integrin that blocks activated lymphocyte adhesion 
and migration into the CNS. It was first approved for treatment of adult RRMS in 2004 but withdrawn from the market in 2006 over reports of PML in patients with MS and Crohn disease treated during the phase III clinical trials [57-59]. Caused by oligodendrocyte infection by neurotropically mutated John Cunningham virus (JCV), PML is a rare brain infection that occurs in immunosuppressed patients [57-59]. Symptoms of PML can resemble MS relapses but are typically subacute in presentation, and while reversible in many cases following cessation of the immunosuppressive agent, PML carries a mortality rate above $30 \%$ and often results in permanent neurologic disability. There have been $>600$ cases of PML reported in adult patients treated with natalizumab to date; there have been no PML cases yet reported in natalizumab-treated patients with POMS. Low or high PML risk is based on the presence or absence of JCV antibodies and titer, use of a previous immunosuppressive agent, and length of natalizumab treatment. Riskmitigation strategies have been implemented, including frequent (every 3 months) serologic testing for JCV antibodies, and screening MRIs every 3 to 6 months in JCV-positive and JCVnegative patients receiving natalizumab, respectively. Additionally, treatment centers, physicians, nurses, pharmacies, and patients are enrolled into a nationwide monitoring program [60]. Despite carrying the greatest risk for PML among MS DMTs, natalizumab is highly efficacious, typically well tolerated, and appears superior to other DMTs in models of long-term morbidity and mortality [61].

Since its approval for adults there have been several open label and retrospective studies evaluating the safety and efficacy of natalizumab in POMS [62-66]. A 2008 case series reported that 3 patients who had both side effects and breakthrough clinical and radiographic disease on one of the injectable therapies tolerated natalizumab well and had no evidence of clinical or radiographic disease activity up to 15 months [64]. A subsequent open-label study followed 19 patients with POMS prospectively. All patients tolerated natalizumab well and remained relapse free. MRI activity was significantly reduced, with only 3 patients developing new T2 lesions at 6 months and no subsequent T2 hyperintense or gadolinium-enhancing lesions thereafter. This study provided class IV evidence for the use of natalizumab in patients with POMS [65]. The most recent and largest prospectively followed cohort study again demonstrated natalizumab was well tolerated and provided excellent suppression of clinical and radiographic disease activity. This study not only included patients who had failed a prior injectable therapy, but also included several patients who were treatment naïve [63]. There were 2 other retrospective studies published in the same year (2013) that provided further evidence that natalizumab is a safe and highly effective treatment in POMS $[62,66]$. Recently 2 Biogensponsored studies of natalizumab safety and tolerability (NCT02137109) and pharmacokinetic and pharmacodynamics (NCT01884935) in children were completed but no results have been reported at the time of writing.
Ocrelizumab is a humanized anti-CD20 monoclonal antibody recently approved by the FDA for both relapsing forms and primary progressive MS in patients aged 18 years and older. Approval was based on 2 phase III studies comparing ocrelizumab with active comparator (IFN) in adult RRMS (OPERA I and OPERA II) and a placebo-controlled trial in progressive MS (ORATORIO) [67, 68]. Ocrelizumab showed high efficacy in reducing symptoms or markers of inflammatory disease (clinical relapses, new or enlarging T2 lesions, and new gadolinium-enhancing T1 lesions) in the OPERA studies, and reduced disability progression in ORATORIO. Infusion reactions, mildly increased risk for infection, and a slightly increased risk for neoplasms were reported in both studies. There is no published experience with this medication in pediatric patients. There is a planned clinical trial for ocrelizumab in pediatric patients, but it is not yet enrolling. However, based on its very high efficacy, tolerability, and relative safety, this will likely be a useful treatment for POMS.

Rituximab is a chimeric anti-CD20 monoclonal antibody that is not approved by the FDA for use in either adult MS or POMS. However, there have been 2 randomized clinical trials in adults $[69,70]$ and 2 retrospective studies in pediatric populations [71, 72]. In adults, the studies demonstrated a significant reduction in relapse rate and MRI activity. The smaller of the pediatric studies included both patients with neuromyelitis optica and 3 with POMS. The average age of the patients with POMS who received rituximab was 15.5 years. The dose used was 208 to $584 \mathrm{mg} / \mathrm{m}^{2}$ (maximum dose $1000 \mathrm{mg}$ per infusion, total dose per cycle range, $416-1168 \mathrm{mg} / \mathrm{m}^{2}$ ), which was administered in 2 doses, 2 weeks apart. The patients with MS had all been on 2 to 3 prior DMTs. They experienced no adverse events and 2 of 3 patients had no relapses. A larger multicenter retrospective cohort of 144 children with several CNS autoimmune conditions included 4 patients with POMS [71]. The median age for the entire cohort was 8 years and all tolerated rituximab well. A subgroup of children $<5$ years ( 46 patients) had no increase in side effects, except a possible trend toward risk of hypogammaglobulinemia. While highly efficacious across neuroimmunologic conditions, there were mixed results in these 4 patients with POMS.

Alemtuzumab is a humanized monoclonal antibody specific for CD52, which depletes circulating lymphocytes including T cells, B cells, natural killer cells, monocytes, and dendritic cells. Upon recovery of lymphocyte populations, there is a presumed shift to $\mathrm{T}$ helper 2 cells and reduction of proinflammatory cells and cytokines [73, 74]. Alemtuzumab was approved in 2014 for adult RRMS after the completion of 2 phase III trials, which reported a significant reduction in relapse rate, new T2 lesions, and gadolinium-enhancing lesions compared with an active IFN comparator $[75,76]$. To date there have been no studies in patients with POMS, though the safety and efficacy of alemtuzumab has been demonstrated in preventing rejection in solid organ and hematopoietic stem cell transplants [77-79]. 
However, alemtuzumab has a high incidence of autoimmune side effects, including $>30 \%$ risk of autoimmune thyroid disease, an approximately $2 \%$ risk of autoimmune thrombocytopenia, and approximately $1 \%$ risk of antibasement membrane glomerulonephritis. There have also been rare lymphoproliferative disease, thyroid cancer, and melanoma reported. Despite these associated side effect profiles, alemtuzumab achieves higher rates of disability-progression free survival and annualized relapse rate reduction at 5 years than IFN, and showed significant benefit in newly diagnosed, treatment naïve patients in phase III clinical trials. Thus, alemtuzumab may be higher risk for use in patients with POMS, but for those with highly active POMS, this may be an appropriate DMT to consider.

\section{Other Agents}

Cyclophosphamide is an alkylating agent that has been used in more aggressive forms of adult RRMS. It is used in other pediatric autoimmune diseases and cancers, though its toxicity and risk for future cancers limits its use in children. A retrospective review of 17 children demonstrated a reduction in relapse rate and stabilization of disability scores at 1 year post-treatment in the majority of patients [80]. However, side effects were frequent, including vomiting, transient alopecia, osteoporosis, and amenorrhea, and at least 1 patient developed bladder cancer. In cases of fulminant demyelination or highly active breakthrough disease activity, cyclophosphamide may be needed to halt the disease while considering other DMTs for ongoing therapy.

\section{Treatment Strategy}

As described in the previous section there is evidence for the use of several MS DMTs in POMS, but this evidence is limited. Most treatment algorithms are based on expert consensus, the adult literature, and clinical experience of the treating physician. Over the last decade, adult treatment algorithms have been re-evaluated, reflecting the growing number of treatment options, many of which appear more efficacious compared to earlier DMT generations [61]. Many specialists in adult MS are now holding DMTs to a higher standard with the goal of achieving NEDA [81]. The rationale supporting NEDA as a clinical outcome evolved from prior observations that early treatment with DMT altered the natural history of MS and delayed disability milestones [82]. Thus, the absence of detectable disease activity could prevent or minimize disability progression. However, NEDA is difficult to sustain across long epochs, even with high-efficacy DMTs [83]. To achieve NEDA many adult neurologists begin treatment with higher efficacy DMTs at disease onset as opposed to switching to one of these agents once a patient has breakthrough disease. The counter argument to starting highly effective DMTs early is the increased risk often associated with these treatments.
Thus, when making treatment decisions, patients and clinicians must weigh the risks inherent to ongoing MS disease activity with those inherent to different treatment options.

The debate over treatment approaches currently focuses on escalation versus early high-efficacy strategies [84]. In their 2011 review, Banwell et al. [85] suggested 2 different treatment models: 1) "start low and escalate if needed"; 2) "start strong, maintain remission". Current POMS treatment recommendations reflect the first model, an escalation algorithm: treatments should be started early, at the time of POMS diagnosis, using lowerefficacy injectable therapies, and patients should be followed closely with clinical evaluations every 3 to 6 months and MRI evaluations every 6 to 12 months [86-88]. If there is evidence of inadequate treatment response as defined by clinical or radiographic disease activity despite compliant use for at least 6 months, 2 or more clinical or radiographic relapses in 12 months, or intolerable medication side effects then a "lateral" treatment switch should be attempted, i.e., switching from glatiramer to an IFN or vice versa. Similar surveillance should be undertaken with the second injectable treatment, and should there be inadequate treatment response or intolerable side effects only then should treatment be "escalated" by switching to a higher-efficacy oral or infusion therapy $[86,89]$. This approach is supported by multiple small, open-label prospective and retrospective studies collectively describing over 600 patients with POMS. Using standard injectable DMTs, patients with POMS demonstrate an average of $30 \%$ reduction in annualized relapse rate between pre- and post-treatment periods [86]. However, treatment failure and discontinuation rates ranged from $25 \%$ to $64 \%$ across studies. Thus, the current consensus for first-line POMS treatment is based on a moderate number of patients, reflects moderate DMT efficacy, and emphasizes medication safety profiles.

Although there is clear evidence to support the current treatment model for POMS there are several reasons to consider implementing higher efficacy therapies early. In retrospective studies, as many as $30 \%$ of POMS patients will demonstrate breakthrough disease activity in the first year of treatment, whereas $44 \%$ opt to switch DMT owing to intolerable side effects [89]. Nonadherence to injectable therapies is high among patients with POMS, likely under-reported, and potentially contributes to inadequate treatment responses [84]. Based on current recommendations, patients with POMS who experience inadequate treatment responses to injectable DMTs could wait 12 to 24 months before being switched to higher-potency DMTs. During this period, patients are at risk for both transient and permanent neurologic deficits, and may experience side effects from acute treatment with high-dose intravenous steroids. Further, clinical relapses have negative impacts on school attendance, academic and athletic performance, lost productivity for caretakers who may need time off from work, as well as negative social consequences [90].

By contrast, the second treatment of Banwell et al. [85] reflects the authors' current clinical practice, and we argue this 
approach should be the standard of care for POMS. Although there have been no studies directly comparing different strategies for first-line therapies a recent systematic review compared clinical outcomes when high-efficacy DMTs were implemented early versus late in adult MS treatment. In aggregate, early initiation of high-efficacy DMTs in adult patients with MS resulted in greater suppression of clinical disease activity, though the MRI and disability progression data were less robust [91]. These findings reflect the authors' clinical experiences treating adult patients with MS, and reflect our current clinical practice in pediatric and adult patients with MS.

Further, we believe efficacy is tied to issues of DMT adherence and noncompliance. The conventional first-line agents are injection therapies, which many patients have difficulty taking regularly. Three recent studies in POMS reported noncompliance rates of $37 \%$ to $47 \%$ [92-94]. In POMS, selfadministered DMT nonadherence is most commonly related to forgetting one's medicine, both for patients and their parents. Further, self-reported physical functioning and parental involvement may play a counterintuitive role, with relapse freedom in the last year correlating with increased risk of nonadherence in at least 1 study. Conversely, a patient's perception of her physical level of functioning was also found to promote adherence [94]. This study reported that the level of parental involvement is inversely related to the parent's perception of his or her child's function. Interestingly parental involvement was shown to be less associated with oral DMTs, which are easier to administer than injections, and may promote patient autonomy [94]. When treatment adherence is poor, DMT efficacy is necessarily reduced; thus, barriers to DMT adherence influence treatment choices [95]. Compliance with directly observed infusion medications is more easily assessed because they require patients to come to a clinic, infusion center, or hospital to receive treatment. This increases contact with a treatment team and may provide increased support and help patients and their parents remember treatment visits. There are complicating factors to consider with infusions, including accessibility and availability of infusion centers, and, in some cases, increased clinical, laboratory, and imaging monitoring. Ultimately, infusion therapy adherence is binary: patients either receive the infusion or they do not. In either case, treatment efficacy is not blurred by questions of compliance.

Our proposed treatment strategy will be called the "highest efficacy early treatment", or HEET, strategy, which advocates starting treatment with the most efficacious DMT suitable to a specific patient. The goal of HEET is to stop disease activity early to prevent deleterious neurodevelopmental deficits now, and disability progression in the future. We feel the perceived safety of lower-efficacy injectable DMTs inadequately considers the unique aspects of POMS addressed in the previous sections, including early tissue level inflammation and axon loss, increased relapse rate, and earlier age of disability suffered by patients with POMS. In choosing a first-line therapy, HEET considers several patient-related factors: pubertal status, JCV status, parent/patient risk aversion, DMT route of administration, and noncompliance risk.

\section{HEET: Prepubertal Patients}

As previously outlined there are several aspects of prepubertal patients, including unique clinical presentations, imaging findings, and CSF profiles, that suggest a different treatment approach might be warranted. Additionally, since all of the utilized medications are only approved in adults most do not have the ability to adjust the dose for weight, which is a significant consideration when treating a population undergoing potentially rapid growth.

For prepubertal patients, we advocate starting with rituximab using $500 \mathrm{mg}$ up to $750 \mathrm{mg}$ per meter squared of body surface area (using the Haycock calculation [96]), with a maximum dose of $1000 \mathrm{mg}$ per dose, dosed at day 1, day 15, and then every 6 months thereafter. Other anti-B-cell monoclonal antibodies are commercially available, including the recently FDA-approved ocrelizumab. However, at this time weightbased dosing of ocrelizumab for pediatric patients is not available, though a phase II clinical trial in patients with POMS is imminent at the time of writing. Thus, future iterations of the HEET strategy may include other anti-B-cell therapies, but the current approach will use rituximab. Additionally, there is a wealth of evidence for the use of rituximab in the pediatric population, including for other autoimmune conditions [97]. For breakthrough disease activity lymphocyte profiling should be considered to monitor for reconstitution of the CD19-positive population, and either the dose should be maximized to $750 \mathrm{mg}$ per meter squared or treatment intervals should be shortened to every 4 months as needed. Prerituximab safety monitoring should include lymphocyte profiles and immunoglobulin $\operatorname{IgM}, \operatorname{IgG}$, and $\operatorname{IgA}$ levels, screening for evidence of vaccination and/or presence of chronic infectious diseases, including HIV, tuberculosis, syphilis, hepatitis B and C, varicella zoster virus, and, if necessary, measles, mumps, and rubella. Safety monitoring should include periodic tuberculosis testing for high-risk patients, and immunoglobulin levels should be screened every 6 months; if levels are low, supplemental intravenous or subcutaneous immunoglobulins can be administered. The long-term effects of B-cell depletion are not yet known, particularly in pediatric patients, and questions remain regarding the appropriate duration of rituximab treatment in patients with POMS. Prospective studies may help systematically answer these questions, but at this time decisions regarding treatment duration will need to be made on an individual basis. For patients with highly active POMS, it is the authors' contention that the known risks of POMS outweigh the potential or unknown risks of long-term B-cell depletion. 
If rituximab is not available, contraindicated, or parental risk aversion prevents its use in the prepubertal child, then second-choice agents will depend on JCV status. For JCVnegative prepubertal patients, natalizumab should be considered. At this time natalizumab per $\mathrm{kg}$ body weight dosing in children has not been established. However, the phase II trials of natalizumab in patients with MS demonstrated similar safety, tolerability, and efficacy between $3 \mathrm{mg}$ and $6 \mathrm{mg}$ per $\mathrm{kg}$ body weight doses [98], while the $3 \mathrm{mg}$ per $\mathrm{kg}$ dose was found to have similar pharmacokinetics in adolescent (aged 12-17 years) patients with Crohn disease to adults [99]. Thus, for JCV negative prepubertal patients, natalizumab $3 \mathrm{mg}$ per $\mathrm{kg}$ body weight given intravenously every 28 days can be considered; for prepubertal patients $>50 \mathrm{~kg}$ we recommend using the full adult dose of $300 \mathrm{mg}$ per dose $(\leq 6 \mathrm{mg} / \mathrm{kg})$. Extended dosing intervals to every 6 weeks could also be considered, though there are no data available on $\alpha 4$-integrin receptor saturation levels at this dosing interval in pediatric patients [100]. Thus, close clinical and radiographic monitoring will be needed to assess treatment efficacy of natalizumab in prepubertal patients, similar to adult patients. Safety monitoring for pediatric patients should parallel that of adult patients, including enrollment in the company-sponsored risk evaluation and mitigation strategy program, JCV antibody testing every 3 months, liver enzyme testing every 3 months, and checking for antinatalizumab antibodies after 6 months of treatment or if clinical or radiographic disease activity is detected. An "exit strategy" should be prepared in advance for all natalizumab-treated patients for the eventuality they seroconvert to JCV positive while receiving natalizumab. If they have received natalizumab for $<2$ years, then treatment should be stopped at 2 years, whereas patients receiving natalizumab for $>2$ years should be stopped and switched to another high-efficacy treatment within 8 weeks to avoid rebound MS disease activity [101]. Clinical and radiographic monitoring for PML should persist for 6 months following natalizumab cessation.

For JCV-positive prepubertal patients opposed to, or unable to receive, infusion therapy, high-efficacy treatment options are relatively limited. There are not enough clinical trials or case series to make recommendations for the oral DMTs in prepubertal patients with POMS. The efficacy of teriflunomide in adults is similar to IFNs [102], and lower than fingolimod or dimethyl fumarate [103], and is associated with severe hepatotoxicity. Thus, the risk/benefit ratio for teriflunomide is not favorable for pediatric patients and we recommend against its use, pending completion of current clinical trials. Compared with dimethyl fumarate, fingolimod likely offers the best combination of efficacy, tolerability, and compliance (owing to once-daily dosing) in prepubertal patients who can swallow pills. However, first-dose bradycardic effects and the risk for rare but serious cardiovascular side effects, PML, and rebound MS disease activity following fingolimod cessation must be considered before use in a prepubertal POMS population. In contrast to the once-daily dosing for fingolimod and teriflunomide, dimethyl fumarate is dosed twice daily, which likely contributes to decreased compliance. Additionally, dimethyl fumarate's associated gastrointestinal side effects, the potential need for aspirin pretreatment, associated PML risk, and potential for sustained lymphopenia all limit dimethyl fumarate's use in prepubertal patients. Thus, in prepubertal patients with POMS for whom rituximab or natalizumab are not options, clinicians should consider following current POMS treatment guidelines. In these cases we recommend minimizing injections by starting with either a once-weekly IFN- $\beta 1$ a intramuscular injection, every other week pegylated-IFN- $\beta 1$ a subcutaneous injection, or 3 times per week glatiramer acetate.

For prepubertal patients with highly active, fulminant demyelinating events, aggressive anti-inflammatory therapy may be required, particularly if they continue to have frequent breakthrough disease with HEET, or recurrent disease upon steroid taper or withdrawal. In these cases, cyclophosphamide induction therapy ( $600 \mathrm{mg} / \mathrm{m}^{2}$ daily for 5 days) may be needed to shut down disease activity while considering the next DMT. Standard-dose cyclophosphamide pulse therapy can also be considered, using $800 \mathrm{mg} / \mathrm{m}^{2}$ monthly for up to 6 months. However, both induction and monthly pulse therapy with cyclophosphamide carry risk for short- and long-term side effects, including serious infectious, hematologic, reproductive, urologic, and neoplastic problems; cyclophosphamide also increases risk for PML with future natalizumab use $[80,104]$. Owing to the potentially significant short- and long-term consequences of this drug it should only be considered in patients refractory to, or contraindicated from, all of the previously mentioned options.

\section{HEET: Postpubertal Patients}

Postpubertal patients ought to be treated similar to adult patients, with some key differences. Directly observed therapies are strongly recommended for adolescents in whom noncompliance is a great concern. For JCV-negative patients $>50 \mathrm{~kg}$, we strongly advocate beginning treatment with natalizumab $300 \mathrm{mg}$ intravenously every 28 days; for patients $<50 \mathrm{~kg}$ the 3 $\mathrm{mg} / \mathrm{kg}$ body weight dose should still be used. This recommendation is based on several factors. First, compared with rituximab, natalizumab is an FDA-approved DMT and could potentially continue to be utilized on-label after a patient's eighteenth birthday. Second, natalizumab offers the best benefitto-risk ratio in JCV-negative patients, with a significant drop off upon seroconversion to JCV positivity. Third, with advancing age the risk for conversion to JCV-positive status increases, with estimates of JCV positivity among patients with POMS ranging from $33 \%$ to as high as $50 \%$ [105, 106]. Thus, delaying treatment with natalizumab increases 
the risk that a patient with POMS will seroconvert to JCV positive and no longer be appropriate for natalizumab treatment. If a JCV-negative patient with POMS becomes JCV positive, treatment should be limited to 2 years, but DMT switch should be planned for no later than 8 weeks following natalizumab cessation to avoid rebound MS disease activity [101].

For JCV-positive postpubertal patients, or for those unable to or unwilling to receive natalizumab, anti-B-cell therapy with either rituximab or ocrelizumab should be considered. If a postpubertal child weighs $<50 \mathrm{~kg}$, then rituximab would likely be a better choice for weight-based dosing, until ocrelizumab dosing is adequately studied in children. Similar pretreatment laboratory tests listed above should be obtained prior to starting anti-B-cell therapy.

For JCV positive postpubertal patients, or those resistant to infusion therapy, then oral DMTs fingolimod or dimethyl fumarate can be considered; we rarely recommend teriflunomide in this age group over concerns of modest efficacy, hepatotoxicity, and teratogenicity. There are no head-tohead clinical superiority trials between fingolimod or dimethyl fumarate, but observational studies and statistical modeling have attempted to address this question. While clinically no differences were identified, dimethyl fumarate was associated with increased odds of gadolinium-enhancing lesions, earlier time to discontinuation, and decreased time to relapse versus fingolimod [107]. Indirect comparisons between oral DMTs and their likelihood of achieving NEDA in a phase III clinical trial demonstrated fingolimod treatment to be more likely to achieve NEDA than dimethyl fumarate or teriflunomide [108]. However, another study found similar numbers needed to treat to prevent relapses, relapses requiring treatment, and disability [109]. While modest, these findings support our preference to start fingolimod over dimethyl fumarate. Fingolimod offers once-daily dosing and relative tolerability, making compliance more likely in patients with POMS. Patients should be screened for antivaricella zoster virus immunoglobulin, and if they are negative vaccination (2 vaccination doses administered 4 weeks apart) should be completed at least 4 weeks before treatment onset. If patients are not amenable to fingolimod then dimethyl fumarate can be started, but we recommend a slow titration as follows: $120 \mathrm{mg}$ daily for 1 week, then $120 \mathrm{mg}$ twice daily for 1 week, then $120 \mathrm{mg}$ in the morning and $240 \mathrm{mg}$ in the evening for 1 week, then $240 \mathrm{mg}$ twice daily. For postpubertal, sexually abstinent males with mild MS disease activity, teriflunomide can be considered if they are considerably needle phobic. Similar to prepubertal patients, and adult patients, for those postpubertal patients who are themselves, or whose parents are, resistant to infusion or oral therapies, we again recommend IFN- $\beta 1$ a once-weekly intramuscular injection or every-other-week pegylated-IFN- $\beta 1$ a subcutaneous injections, or glatiramer acetate 3 times weekly injections.

\section{Conclusions}

Despite evidence demonstrating increased inflammatory disease activity with early tissue destruction and poorer clinical outcomes in POMS, current treatment guidelines emphasize perceived DMT safety over efficacy, promoting injectable IFNs or glatiramer acetate as first-line therapies. While considered safe, these DMTs suffer from modest efficacy and compliance issues stemming from route of administration and side effects. Our proposed HEET strategy aims to improve POMS clinical and neurodevelopmental outcomes by preventing permanent neurologic injury known to occur early in POMS. While future studies are needed to determine the safety, efficacy, and tolerability of all current and pipeline DMTs in POMS, we argue high-efficacy treatments ought to be prioritized ahead of lower efficacy treatments. Future prospective trials comparing HEET to current treatment guidelines are needed. In the interim, pediatric neurologists and their patients may benefit by bringing the HEET strategy into their daily clinical practice.

Acknowledgements MP McGinley is supported by National Multiple Sclerosis Society Sylvia Lawry Physician Fellowship FP-1506-04742.

Required Author Forms Disclosure forms provided by the authors are available with the online version of this article.

\section{References}

1. Yeh EA, Chitnis T, Krupp L, et al. Pediatric multiple sclerosis. Nat Rev Neurol 2009;5:621-631.

2. Langer-Gould A, Zhang JL, Chung J, Yeung Y, Waubant E, Yao J. Incidence of acquired CNS demyelinating syndromes in a multiethnic cohort of children. Neurology 2011;77:1143-1148.

3. Krupp LB, Banwell B, Tenembaum S, International Pediatric MS Study Group. Consensus definitions proposed for pediatric multiple sclerosis and related disorders. Neurology 2007;68:S7-12.

4. Tardieu M, Banwell B, Wolinsky JS, Pohl D, Krupp LB. Consensus definitions for pediatric MS and other demyelinating disorders in childhood. Neurology 2016;87:S8-S11.

5. Bavdekar SB. Pediatric clinical trials. Perspect Clin Res 2013;4: 89-99.

6. National Commission for the Protection of Human Subjects of Biome Beha Resea, Ryan KJP. The Belmont Report: Ethical Principles and Guidelines for the Protection of Human Subjects of Research-the National Commission for the Protection of Human Subjects of Biomedical and Behavioral Research: US Government Printing Office; 1978.

7. Polman CH, Reingold SC, Banwell B, et al. Diagnostic criteria for multiple sclerosis: 2010 revisions to the McDonald criteria. Ann Neurol 2011;69:292-302.

8. Sadaka Y, Verhey LH, Shroff MM, et al. 2010 McDonald criteria for diagnosing pediatric multiple sclerosis. Ann Neurol 2012;72: 211-223.

9. Huppke B, Ellenberger D, Rosewich H, Friede T, Gärtner J, Huppke P. Clinical presentation of pediatric multiple sclerosis before puberty. Eur J Neurol 2014;21:441-446. 
10. Banwell B, Arnold DL, Tillema JM, et al. MRI in the evaluation of pediatric multiple sclerosis. Neurology 2016;87:S88-96.

11. Waubant E, Chabas D, Okuda DT, et al. Difference in disease burden and activity in pediatric patients on brain magnetic resonance imaging at time of multiple sclerosis onset vs adults. Arch Neurol 2009;66:967-971.

12. Chabas D, Castillo-Trivino T, Mowry EM, Strober JB, Glenn OA, Waubant E. Vanishing MS T2-bright lesions before puberty: a distinct MRI phenotype? Neurology 2008;71:1090-1093.

13. Tintore M, Rovira A, Rio J, et al. Do oligoclonal bands add information to MRI in first attacks of multiple sclerosis? Neurology 2008; 70:1079-1083.

14. Chabas D, Ness J, Belman A, et al. Younger children with MS have a distinct CSF inflammatory profile at disease onset. Neurology 2010;74:399-405.

15. Renoux C, Vukusic S, Mikaeloff Y, et al. Natural history of multiple sclerosis with childhood onset. N Engl J Med 2007;356: 2603-2613.

16. Amato MP, Goretti B, Ghezzi A, et al. Neuropsychological features in childhood and juvenile multiple sclerosis: five-year follow-up. Neurology 2014;83:1432-1438.

17. Baruch NF, O'Donnell EH, Glanz BI, et al. Cognitive and patientreported outcomes in adults with pediatric-onset multiple sclerosis. Mult Scler 2016;22:354-361.

18. Trojano M, Paolicelli D, Bellacosa A, Fuiani A, Cataldi S, Di Monte E. Atypical forms of multiple sclerosis or different phases of a same disease? Neurol Sci 2004;25:s323-s325.

19. Benson L, Healy B, Gorman M, et al. Elevated relapse rates in pediatric compared to adult MS persist for at least 6 years. Mult Scler Relat Disord 2014;3:186-193.

20. Gorman MP, Healy BC, Polgar-Turcsanyi M, Chitnis T. Increased relapse rate in pediatric-onset compared with adult-onset multiple sclerosis. Arch Neurol 2009;66:54-59.

21. Pfeifenbring S, Bunyan RF, Metz I, et al. Extensive acute axonal damage in pediatric multiple sclerosis lesions. Ann Neurol 2015;77:655-667.

22. Kerbrat A, Aubert-Broche B, Fonov V, et al. Reduced head and brain size for age and disproportionately smaller thalami in childonset MS. Neurology 2012;78:194-201.

23. Aubert-Broche B, Fonov V, Narayanan S, et al. Onset of multiple sclerosis before adulthood leads to failure of age-expected brain growth. Neurology 2014;83:2140-2146.

24. Weier K, Fonov V, Aubert-Broche B, Arnold DL, Banwell B, Collins DL. Impaired growth of the cerebellum in pediatriconset acquired CNS demyelinating disease. Mult Scler 2016;22: 1266-1278

25. Till C, Ghassemi R, Aubert-Broche B, et al. MRI correlates of cognitive impairment in childhood-onset multiple sclerosis. Neuropsychology 2011;25:319-332.

26. Weier K, Till C, Fonov V, et al. Contribution of the cerebellum to cognitive performance in children and adolescents with multiple sclerosis. Mult Scler 2016;22:599-607.

27. Till C, Deotto A, Tipu V, et al. White matter integrity and math performance in pediatric multiple sclerosis: a diffusion tensor imaging study. Neuroreport 2011;22:1005-1009.

28. Rocca MA, Valsasina P, Absinta M, et al. Intranetwork and internetwork functional connectivity abnormalities in pediatric multiple sclerosis. Hum Brain Mapp 2014;35:4180-4192.

29. Charvet LE, Cleary RE, Vazquez K, Belman AL, Krupp LB, US Network for Pediatric MS. Social cognition in pediatric-onset multiple sclerosis (MS). Mult Scler 2014;20:1478-1484.

30. Goretti B, Portaccio E, Ghezzi A, et al. Fatigue and its relationships with cognitive functioning and depression in paediatric multiple sclerosis. Mult Scler 2012;18:329-334.
31. Amato MP, Goretti B, Ghezzi A, et al. Cognitive and psychosocial features in childhood and juvenile MS: two-year follow-up. Neurology 2010;75:1134-1140.

32. Till C, Udler E, Ghassemi R, Narayanan S, Arnold DL, Banwell BL. Factors associated with emotional and behavioral outcomes in adolescents with multiple sclerosis. Mult Scler 2012;18:1170-1180.

33. Ruano L, Branco M, Portaccio E, et al. Patients with paediatriconset multiple sclerosis are at higher risk of cognitive impairment in adulthood: an Italian collaborative study. Mult Scler 2017: 1352458517717341.

34. Balint B, Haas J, Schwarz A, et al. T-cell homeostasis in pediatric multiple sclerosis: old cells in young patients. Neurology 2013;81: 784-792.

35. Kieseier BC. The mechanism of action of interferon- $\beta$ in relapsing multiple sclerosis. CNS Drugs 2011;25:491-502.

36. Ghezzi A, Immunomodulatory Treatment of Early Onset MS (ITEMS) Group. Immunomodulatory treatment of early onset multiple sclerosis: results of an Italian Co-operative Study. Neurol Sci 2005;26:s183-s186.

37. Ghezzi A, Amato MP, Capobianco M, et al. Disease-modifying drugs in childhood-juvenile multiple sclerosis: results of an Italian co-operative study. Mult Scler 2005;11:420-424.

38. Mikaeloff Y, Caridade G, Tardieu M, Suissa S, KIDSEP Study Group of the French Neuropediatric Society. Effectiveness of early beta interferon on the first attack after confirmed multiple sclerosis: a comparative cohort study. Eur J Paediatr Neurol 2008;12:205-209.

39. Mikaeloff $Y$, Moreau T, Debouverie $M$, et al. Interferon- $\beta$ treatment in patients with childhood-onset multiple sclerosis. J Pediatr 2001;139:443-446.

40. Pohl D, Rostasy K, Gartner J, Hanefeld F. Treatment of early onset multiple sclerosis with subcutaneous interferon beta-1a. Neurology 2005;64:888-890.

41. Tenembaum SN, Segura MJ. Interferon beta-1 a treatment in childhood and juvenile-onset multiple sclerosis. Neurology 2006;67: 511-513.

42. Banwell B, Reder AT, Krupp L, et al. Safety and tolerability of interferon beta-1b in pediatric multiple sclerosis. Neurology 2006;66:472-476.

43. Lalive $\mathrm{PH}$, Neuhaus $\mathrm{O}$, Benkhoucha $\mathrm{M}$, et al. Glatiramer acetate in the treatment of multiple sclerosis. CNS Drugs 2011;25:401-414.

44. Kornek B, Bernert G, Balassy C, Geldner J, Prayer D, Feucht M. Glatiramer acetate treatment in patients with childhood and juvenile onset multiple sclerosis. Neuropediatrics 2003;34:120-126.

45. Bielekova B, Catalfamo M, Reichert-Scrivner S, et al. Regulatory CD56(bright) natural killer cells mediate immunomodulatory effects of IL-2Ralpha-targeted therapy (daclizumab) in multiple sclerosis. Proc Natl Acad Sci U S A 2006;103:5941-5946.

46. Gorman MP, Tillema J, Ciliax AM, Guttmann CR, Chitnis T. Daclizumab use in patients with pediatric multiple sclerosis. Arch Neurol 2012;69:78-81.

47. Biogen/Abbvie. Zinbryta prescribing information 05/2017; Available at: https:/www.zinbryta.com/content/dam/commercial/ multiple-sclerosis/zinbryta/pat/en us/pdfs/zinbryta-prescribinginformation.pdf. Accessed June 21, 2017.

48. Pelletier D, Hafler DA. Fingolimod for multiple sclerosis. N Engl J Med 2012;366:339-347.

49. Fragoso YD, Alves-Leon SV, Barreira AA, et al. Fingolimod prescribed for the treatment of multiple sclerosis in patients younger than age 18 years. Pediatr Neurol 2015;53:166-168.

50. Safety and Efficacy of Fingolimod in Pediatric Patients With Multiple Sclerosis. Available at: https://clinicaltrials.gov/ct2/ show/NCT01892722?term=fingolimod\&rank=40. Accessed January 16, 2017.

51. Hatcher SE, Waubant E, Nourbakhsh B, Crabtree-Hartman E, Graves JS. Rebound syndrome in patients with multiple sclerosis after cessation of fingolimod treatment. JAMA Neurol 2016;73:790-794. 
52. Gold R, Linker R, Stangel M. Fumaric acid and its esters: an emerging treatment for multiple sclerosis with antioxidative mechanism of action. Clin Immunol 2012;142:44-48.

53. Makhani N, Schreiner T. Oral dimethyl fumarate in children with multiple sclerosis: a dual-center study. Pediatr Neurol 2016;57: 101-104.

54. Biogen. Phase 3 Efficacy and Safety Study of BG00012 in Pediatric Subjects With Relapsing-remitting Multiple Sclerosis (RRMS) (CONNECT). Available at: https://clinicaltrials.gov/ct2/ show/NCT02283853. Accessed March 27, 2017.

55. Fox RI, Herrmann ML, Frangou CG, et al. Mechanism of action for leflunomide in rheumatoid arthritis. Clin Immunol 1999;93: 198-208.

56. Genzyme ASC. Efficacy, Safety and Pharmacokinetics of Teriflunomide in Pediatric Patients With Relapsing Forms of Multiple Sclerosis (TERIKIDS). 2017; Available at: https:// clinicaltrials.gov/ct2/show/NCT02201108?term= TERIKIDS\&rank=1. Accessed March 27, 2017.

57. Langer-Gould A, Atlas SW, Green AJ, Bollen AW, Pelletier D. Progressive multifocal leukoencephalopathy in a patient treated with natalizumab. N Engl J Med 2005;353:375-381.

58. Kleinschmidt-DeMasters BK, Tyler KL. Progressive multifocal leukoencephalopathy complicating treatment with natalizumab and interferon beta-1a for multiple sclerosis. N Engl J Med 2005;353:369-374.

59. Van Assche G, Van Ranst M, Sciot R, et al. Progressive multifocal leukoencephalopathy after natalizumab therapy for Crohn's disease. N Engl J Med 2005;353:362-368.

60. D'Amico E, Zanghi A, Leone C, Tumani H, Patti F. Treatmentrelated progressive multifocal leukoencephalopathy in multiple sclerosis: a comprehensive review of current evidence and future needs. Drug Saf 2016;39:1163-1174.

61. Bargiela D, Bianchi MT, Westover MB, et al. Selection of first-line therapy in multiple sclerosis using risk-benefit decision analysis. Neurology 2017;88:677-684.

62. Kornek B, Aboul-Enein F, Rostasy K, et al. Natalizumab therapy for highly active pediatric multiple sclerosis. JAMA Neurol 2013;70:469-475.

63. Ghezzi A, Pozzilli C, Grimaldi LM, et al. Natalizumab in pediatric multiple sclerosis: results of a cohort of 55 cases. Mult Scler 2013; 19:1106-1112.

64. Huppke P, Stark W, Zürcher C, Huppke B, Brück W, Gärtner J. Natalizumab use in pediatric multiple sclerosis. Arch Neurol 2008;65:1655-1658.

65. Ghezzi A, Pozzilli C, Grimaldi LM, et al. Safety and efficacy of natalizumab in children with multiple sclerosis. Neurology 2010;75:912-917.

66. Arnal-Garcia C, García-Montero MR, Málaga I, et al. Natalizumab use in pediatric patients with relapsing-remitting multiple sclerosis. Eur J Paediatr Neurol 2013;17:50-54.

67. Montalban X, Hauser SL, Kappos L, et al. Ocrelizumab versus placebo in primary progressive multiple sclerosis. N Engl J Med 2017;376:209-220.

68. Hauser SL, Bar-Or A, Comi G, et al. Ocrelizumab versus interferon beta-1a in relapsing multiple sclerosis. N Engl J Med 2017; 376: 221-234.

69. Hawker K, O'Connor P, Freedman MS, et al. Rituximab in patients with primary progressive multiple sclerosis: results of a randomized double-blind placebo-controlled multicenter trial. Ann Neurol 2009;66:460-471.

70. Hauser SL, Waubant E, Arnold DL, et al. B-cell depletion with rituximab in relapsing-remitting multiple sclerosis. N Engl J Med 2008;358:676-688.

71. Dale RC, Brilot F, Duffy LV, et al. Utility and safety of rituximab in pediatric autoimmune and inflammatory CNS disease. Neurology 2014;83:142-150.
72. Beres SJ, Graves J, Waubant E. Rituximab use in pediatric central demyelinating disease. Pediatr Neurol 2014;51:114-118.

73. Cox AL, Thompson SA, Jones JL, et al. Lymphocyte homeostasis following therapeutic lymphocyte depletion in multiple sclerosis. Eur J Immunol 2005;35:3332-3342.

74. Hu Y, Turner MJ, Shields J, et al. Investigation of the mechanism of action of alemtuzumab in a human CD52 transgenic mouse model. Immunology 2009;128:260-270.

75. Cohen JA, Coles AJ, Arnold DL, et al. Alemtuzumab versus interferon beta 1a as first-line treatment for patients with relapsingremitting multiple sclerosis: a randomised controlled phase 3 trial. Lancet 2012;380:1819-1828.

76. Coles AJ, Twyman CL, Arnold DL, et al. Alemtuzumab for patients with relapsing multiple sclerosis after disease-modifying therapy: a randomised controlled phase 3 trial. Lancet 2012;380: 1829-1839.

77. Tan HP, Donaldson J, Ellis D, et al. Pediatric living donor kidney transplantation under alemtuzumab pretreatment and tacrolimus monotherapy: 4-year experience. Transplantation 2008;86:17251731.

78. Kim IK, Choi J, Vo AA, et al. Safety and efficacy of alemtuzumab induction in highly sensitized pediatric renal transplant recipients. Transplantation (in press).

79. Abdel-Azim H, Mahadeo KM, Zhao Q, et al. Unrelated donor hematopoietic stem cell transplantation for the treatment of nonmalignant genetic diseases: an alemtuzumab based regimen is associated with cure of clinical disease; earlier clearance of alemtuzumab may be associated with graft rejection. Am J Hematol 2015;90:1021-1026.

80. Makhani N, Gorman MP, Branson HM, Stazzone L, Banwell BL, Chitnis T. Cyclophosphamide therapy in pediatric multiple sclerosis. Neurology 2009;72:2076-2082.

81. Bevan CJ, Cree BA. Disease activity free status: a new end point for a new era in multiple sclerosis clinical research? JAMA Neurol 2014;71:269-270.

82. Jeffery DR. Early intervention with immunomodulatory agents in the treatment of multiple sclerosis. J Neurol Sci 2002;197:1-8.

83. Rotstein DL, Healy BC, Malik MT, Chitnis T, Weiner HL. Evaluation of no evidence of disease activity in a 7-year longitudinal multiple sclerosis cohort. JAMA Neurol 2015;72:152-158.

84. Chitnis T, Ghezzi A, Bajer-Kornek B, Boyko A, Giovannoni G, Pohl D. Pediatric multiple sclerosis: Escalation and emerging treatments. Neurology 2016;87:S103-S109.

85. Banwell B, Bar-Or A, Giovannoni G, Dale RC, Tardieu M. Therapies for multiple sclerosis: considerations in the pediatric patient. Nat Rev Neurol 2011;7:109-122.

86. Ghezzi A, Amato MP, Makhani N, Shreiner T, Gartner J, Tenembaum S. Pediatric multiple sclerosis: Conventional firstline treatment and general management. Neurology 2016;87: S97-S102.

87. Ghezzi A, Banwell B, Boyko A, et al. The management of multiple sclerosis in children: a European view. Mult Scler 2010;16: 1258-1267.

88. Chitnis T, Tenembaum S, Banwell B, et al. Consensus statement: evaluation of new and existing therapeutics for pediatric multiple sclerosis. Mult Scler J 2012;18:116-127.

89. Yeh EA, Waubant E, Krupp LB, et al. Multiple sclerosis therapies in pediatric patients with refractory multiple sclerosis. Arch Neurol 2011;68:437-444.

90. Boyd JR, MacMillan LJ. Experiences of children and adolescents living with multiple sclerosis. J Neurosci Nurs 2005;37:334-342.

91. Merkel B, Butzkueven H, Traboulsee AL, Havrdova E, Kalincik T. Timing of high-efficacy therapy in relapsing-remitting multiple sclerosis: a systematic review. Autoimmun Rev 2017;16:658-665. 
92. Thannhauser JE, Mah JK, Metz LM. Adherence of adolescents to multiple sclerosis disease-modifying therapy. Pediatr Neurol 2009;41:119-123.

93. Lulu S, Julian L, Shapiro E, Hudson K, Waubant E. Treatment adherence and transitioning youth in pediatric multiple sclerosis. Mult Scler Relat Disord 2014;3:689-695.

94. Schwartz CE, Grover SA, Powell VE, et al. Risk factors for nonadherence to disease-modifying therapy in pediatric multiple sclerosis. Mult Scler 2017:1352458517695469.

95. Patti F. Optimizing the benefit of multiple sclerosis therapy: the importance of treatment adherence. Patient Prefer Adherence 2010;4:1-9.

96. Haycock GB, Schwartz GJ, Wisotsky DH. Geometric method for measuring body surface area: a height-weight formula validated in infants, children, and adults. J Pediatr 1978;93:62-66.

97. Mahmoud I, Jellouli M, Boukhris I, et al. Efficacy and safety of rituximab in the management of pediatric systemic lupus erythematosus: a systematic review. J Pediatr 2017; 187: 213-219.e2.

98. Miller DH, Khan OA, Sheremata WA, et al. A controlled trial of natalizumab for relapsing multiple sclerosis. N Engl J Med 2003;348:15-23.

99. Hyams JS, Wilson DC, Thomas A, et al. Natalizumab therapy for moderate to severe Crohn disease in adolescents. J Pediatr Gastroenterol Nutr 2007;44:185-191.

100. Zhovtis Ryerson L, Frohman TC, Foley J, et al. Extended interval dosing of natalizumab in multiple sclerosis. J Neurol Neurosurg Psychiatry 2016;87:885-889.

101. Kappos L, Radue EW, Comi G, et al. Switching from natalizumab to fingolimod: A randomized, placebo-controlled study in RRMS. Neurology 2015;85:29-39.
102. Vermersch P, Czlonkowska A, Grimaldi LM, et al. Teriflunomide versus subcutaneous interferon beta- $1 \mathrm{a}$ in patients with relapsing multiple sclerosis: a randomised, controlled phase 3 trial. Mult Scler 2014;20:705-716.

103. Boster A, Nicholas J, Wu N, et al. Comparative effectiveness research of disease-modifying therapies for the management of multiple sclerosis: analysis of a large health insurance claims database. Neurol Ther 2017;6:91-102.

104. Hanumanthe SB, Francisco C, Hart J, Graves J, Waubant E. Biopsy-supported tumefactive demyelination of the central nervous system in children. J Child Neurol 2016;31:1528-1533.

105. Hennes EM, Kornek B, Huppke P, Reindl M, Rostasy K, Berger T. Age-dependent seroprevalence of JCV antibody in children. Neuropediatrics 2016;47:112-114.

106. Huppke P, Hummel H, Ellenberger D, et al. JC virus antibody status in a pediatric multiple sclerosis cohort: prevalence, conversion rate and influence on disease severity. Mult Scler 2015;21: 382-387.

107. Hersh CM, Love TE, Cohn S, et al. Comparative efficacy and discontinuation of dimethyl fumarate and fingolimod in clinical practice at 12-month follow-up. Mult Scler Relat Disord 2016;10: 44-52.

108. Nixon R, Bergvall N, Tomic D, Sfikas N, Cutter G, Giovannoni G. No evidence of disease activity: indirect comparisons of oral therapies for the treatment of relapsing-remitting multiple sclerosis. Adv Ther 2014;31:1134-1154.

109. Freedman MS, Montalban X, Miller AE, et al. Comparing outcomes from clinical studies of oral disease-modifying therapies (dimethyl fumarate, fingolimod, and teriflunomide) in relapsing MS: Assessing absolute differences using a number needed to treat analysis. Mult Scler Relat Disord 2016;10:204-212. 